\title{
Acute and Chronic Ophthalmic Involvement, Severity, and Sequelae in Stevens-Johnson Syndrome and Toxic Epidermal Necrolysis
}

\author{
Sunil Ganekal, Ashwini Nagarajappa \\ Department of Ophthalmology, JJM Medical College, Davanagere, India
}

Purpose: To study the ocular manifestations, its severity and sequelae in patients with Stevens-Johnson syndrome (SJS) and toxic epidermal necrolysis (TEN).

Methods: Prospective study of 44 consecutive patients (30 SJS and 14 TEN) presenting in the acute phase of the disease. Patients were evaluated by dermatologist as well as physician for systemic status, skin lesions and mucosal involvement. Detailed history taking, visual acuity, ophthalmic evaluation (lid margin, corneal, conjunctival changes, tear film and ocular surface). Ocular severity score (OSS) was assessed at baseline (acute) and at 6 months (chronic / OSS6), graded as mild, moderate and severe.

Results: Mean age was $28.15 \pm 15.78$ years. Sixty-five eyes of 33 patients were included for final analysis. Thirty-eight patients (86.4\%) had ocular manifestations. Drugs were the most common causative factor (95.4\%). At base line mild, moderate, and severe OSS was seen in 43.1\%, 44.6\%, and 12.3\% eyes. At 6 months mild, moderate, and severe OSS was seen in $44.6 \%, 7.7 \%$, and $6.2 \%$ of eyes. There was a significant correlation between age of the patient and OSS at 6 months $(p=0.02)$. Younger age had higher chronic OSS. Patients with TEN had higher acute $(p=0.001)$ and chronic $(p=0.001)$ OSS than SJS. Three mucosal surface involvement associated with higher acute and chronic OSS ( $p=0.001)$. No long-term ocular complications observed in 27 / 65 (41.5\%) eyes. Acute OSS correlated significantly with chronic OSS, at 1 and 6 months ( $p=0.001)$.

Conclusions: Greater severity of the disease, more number of mucosal surfaces involved and shorter symptom lag correlated with more severe acute and chronic ocular manifestations. The severity of lid margin involvement and corneal involvement in acute stage were good predictors of severity of chronic ocular findings. Initial severity of ocular involvement correlated with severity of ocular sequelae.

Key Words: Ocular manifestations, Ocular severity score, Stevens-Johnson syndrome

Received: September 17, 2020 Final revision: October 27, 2020 Accepted: December 11, 2020

Corresponding Author: Sunil Ganekal, FRCS. Department of Ophthalmology, JJM Medical College, Medical College Rd, MCC B Block, Kuvempu Nagar, Davangere 577004, India. Tel: 91-8192-222565, Fax: 918192-222565, E-mail: drgsunil@yahoo.com
Stevens-Johnson syndrome (SJS) and its more severe variant toxic epidermal necrolysis (TEN) are acute inflammatory disorders that affect the skin and mucous membranes. These disorders can affect any age group and occur usually as a consequence of adverse drug reactions. A va- 
riety of drugs including antibiotics, nonsteroidal anti-inflammatory drugs, antiepileptics have been implicated. The characteristic features are skin tenderness, erythema of the skin which is followed by cutaneous and mucosal sloughing of varying severity and extent. These disorders are life-threatening due to multisystem involvement and mortality rates are high. Ocular complications occur in both the acute and the chronic stage and are extremely difficult to treat. In the acute stage, there is extensive inflammation of the ocular surface with formation of pseudomembranes, corneal and/or conjunctival epithelial defects. Loss of limbal stem cells occurs, as a result of which, the corneal epithelium fails to regenerate. This results in conjunctival epithelial invasion into the cornea (conjunctivalization) and cicatricial changes of the ocular surface in the chronic stage [1].

Most patients with SJS/TEN consult a physician/dermatologist in the acute stage. Since attention is mainly directed to the maintenance of vital functions and stabilising the patient, the ocular management is often compromised. Often, patients seek ocular treatment when the disease is in the chronic stage. This chronic stage is characterized by persistent epithelial defects, ulceration, and perforation, resulting in corneal cicatricial changes such as neovascularization, opacification, keratinization, and symblepharon [2].

The other issue that could compound the ocular involvement further is the absence of standardized ophthalmologic treatment for the prevention of ocular complications [1]. It is agreed upon however that in the critical acute phase, early and local aggressive measures play an important role in preventing or minimizing the severity of long-term ocular complications [3]. As more than $60 \%$ develop ocular involvement in the acute stage, an ophthalmologist consultation is mandatory as soon as diagnosis is made. Also, patients with ocular involvement should undergo frequent ophthalmic follow-up examinations to ensure the best possible outcome [3]. Hence we took up this study to analyze the ocular manifestations and its sequelae in SJS ad TEN.

\section{Materials and Methods}

Prospective observational study of 44 consecutive patients (30 SJS and 14 TEN) presenting in the acute phase (within 2 weeks) of the disease were included in the study. Informed consent was taken from all patients and the study was approved by the Institutional review board. The dermatological diagnosis of SJS/TEN was made based on presence of a serious mucocutaneous illness with characteristic macules or flat atypical target lesions with epidermal detachment involving $<10 \%$ body surface area in SJS and $>30 \%$ body surface area in TEN associated with a prominent acute prodromal period and involvement of at least two mucosal sites.

Patients presenting after 2 weeks from the onset of symptoms, history of ocular surface surgery, long-term topical eye medications, pre-existing dry eye, any intraocular surgery within the past 3 months, ocular cicatricial pemphigoid/pemphigus vulgaris, contact dermatitis, chemical, thermal, radiation and infections affecting ocular sur-
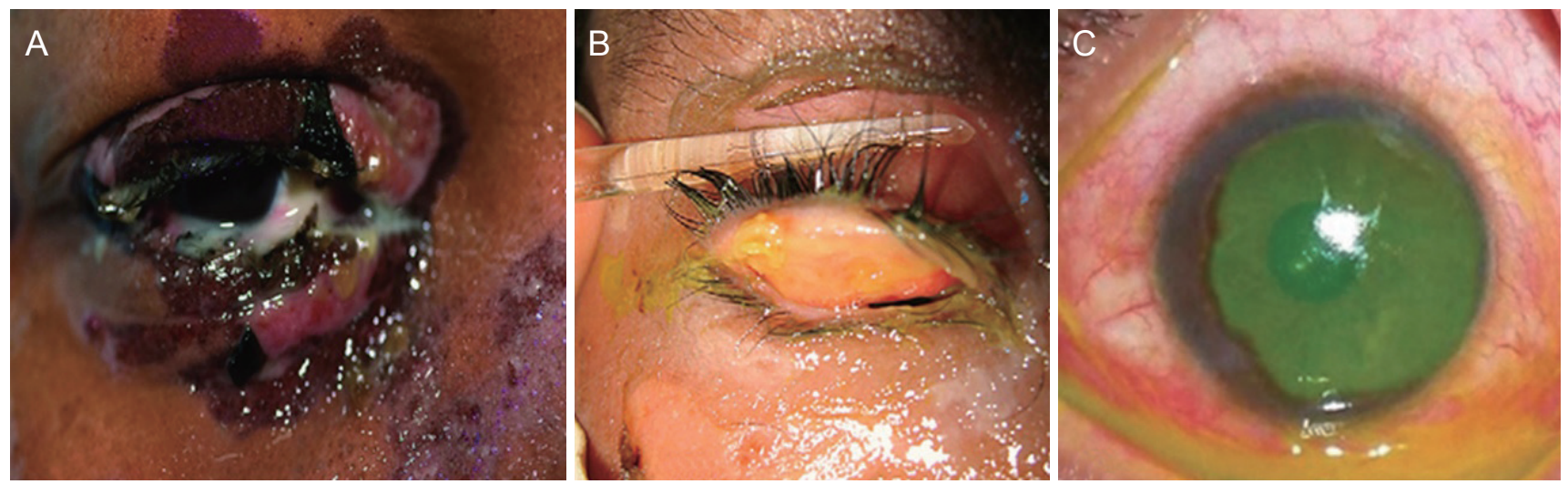

Fig. 1. (A) Ocular involvement in patient with toxic epidermal necrolysis, (B) Stevens- Johnson syndrome patient with conjunctival pseudomembrane, and (C) flourescein staining of the cornea. Informed consent for publication of the clinical images was obtained from the patient. 
face were excluded from the study. The demographic details of the patients were recorded. In all cases, a detailed clinical history pertaining to recent drug use, infections, onset of symptoms and past history of systemic illness was sought. General physical examination with emphasis on noting the extent of mucosal and skin involvement was done. The dermatological diagnosis of SJS/TEN was established according to the inclusion criteria described above. Initial ocular examination was done at the bedside (Fig. 1A-1C). Topical anesthetic eye drops (proparacaine) was used to facilitate the ocular examination. Visual acuity was tested at the initial examination using an illuminated Snellen's chart, with the patient seated at a distance of $6 \mathrm{~m}$. As soon as the patient was fit, a detailed ocular examination was done including slit lamp examination, fluorescein staining of the cornea and fundus evaluation. All patients were reviewed daily while in the hospital. At the time of discharge from the hospital, patients were advised to follow up every week for a month and thereafter every month for 6 months. At 1-month follow-up, best-corrected visual acuity was recorded and tear function tests including tear film break-up time (TFBUT), Schirmer test (basal) and vital staining (using fluorescien and rose bengal) were done. Main outcome measure was presence of ocular sequelae like trichiasis, ocular surface disorders like dry eyes, stem cell deficiency (conjunctivalization of the corneal surface, neovascularization, chronic inflammation and recurrent or persistent corneal epithelial defects).

We classified the ocular findings in the acute stage according to a severity grading system which we adapted and modified from that developed by Sotozono et al. [4] as follows: severity grading of lid margin (muco-cutaneous junction, MCJ) involvement graded as grade 0 , normal lid margin; grade 1, erythema and edema of lid margin with no ulceration; grade 2, ulceration of less than one half of the lid margin; grade 3, ulceration of more than half the lid margin. Severity grading of conjunctival hyperemia graded as grade 0 , absence of hyperemia; grade 1, mild (minimal or sectoral engorgement of the conjunctival vessels); grade 2, moderate (diffuse engorgement of the conjunctival vessels); grade 3, severe hyperemia (significant engorgement of the conjunctival vessels). Severity grading of conjunctival inflammatory adhesions graded as grade 0 , no inflammatory adhesions; grade 1 , inflammatory pseudo-membrane; grade 2, inflammatory adhesions which can be broken; grade 3, symblepharon of any extent. Severity

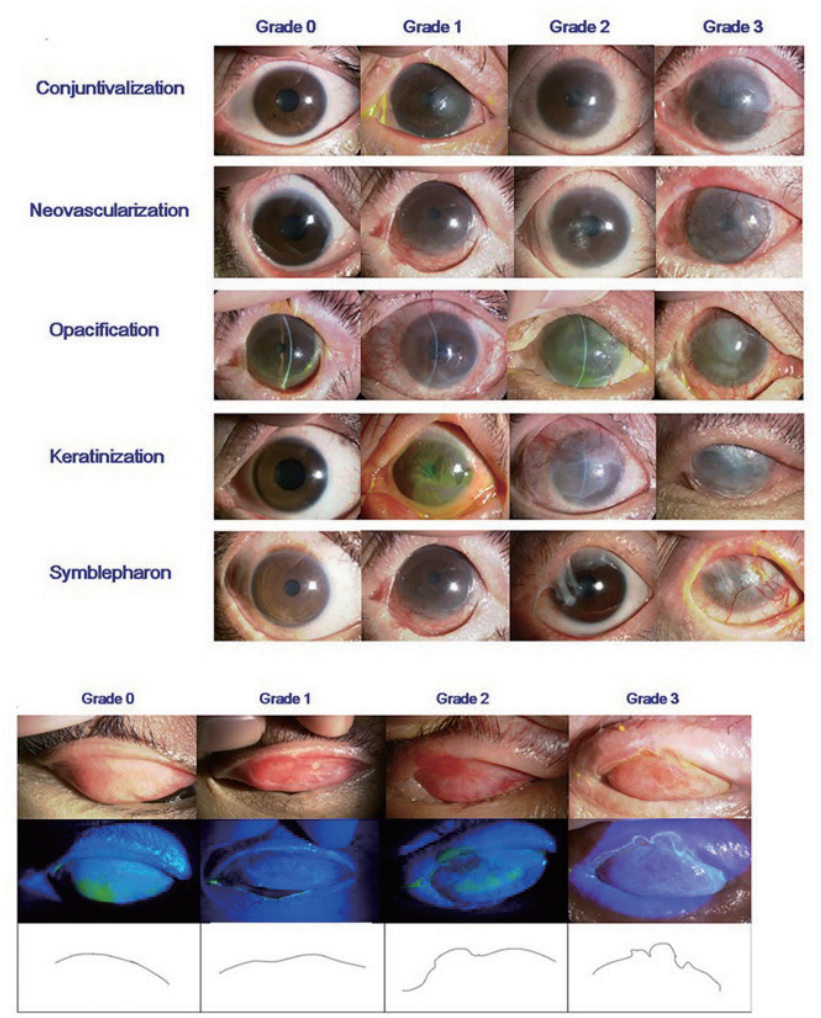

Fig. 2. Bilateral healed corneal perforation and corneal opacity with vascularization. Standard photos of ocular surface grading scores in Stevens-Johnson syndrome and toxic epidermal necrolysis.

grading of corneal epithelial lesions graded as grade 0 , no punctate staining; grade 1, corneal staining involving less than half of the cornea/focal staining; grade 2, corneal staining involving more than half of the cornea/diffuse staining; grade 3, corneal epithelial defect) (Fig. 2).

The severity grading of ocular findings in the chronic stage was adapted (after some modification) from the grading system for chronic ocular complications developed by Sotozono et al. [4]. Severity grading of lid margin (MCJ) involvement graded as grade 0 , normal MCJ; grade 1 , mild irregularity of MCJ; grade 2, moderate irregularity of MCJ; grade 3, severe irregularity of MCJ. Trichiasis graded as grade 0 , no trichiasis; grade 1, trichiasis present. Meibomian gland involvement graded grade 0 , absent (clear oily fluid expressed); grade 1, present (yellowish-white oily fluid/cheesy material expressed). Punctal involvement graded as grade 0 , normal patent puncta; grade 1 , punctal scarring present. Symblepharon formation graded as grade 0 , no symblepharon; grade 1, symblepharon present. Severity grading of corneal epithelial lesions grad- 
ed as grade 0 , no punctate staining; grade 1, corneal staining involving less than half of the cornea/focal staining; grade 2, corneal staining involving more than half of the cornea/diffuse staining; grade 3, corneal epithelial defect. Corneal neovascularization graded as grade 0 , no neovascularization; grade 1, neovascularization confined to the corneal periphery; grade 2, neovascularization extending up to the central cornea. Corneal opacification graded as grade 0 , clear cornea with iris details clearly visualized; grade 1, partial obscuration of the iris details; grade 2, iris details poorly seen with pupil margin just visible; grade 3 , complete obscuration of iris and pupil details. Tear function tests were performed at 6-month follow-up. These included Schirmer test with anesthetic; value of $<10 \mathrm{~mm}$ at 5 minutes was considered abnormal. TFBUT $<10$ seconds was considered abnormal rose bengal test; a score of $>3$ according to van Bijsterveld's scoring system [5] was considered abnormal.

For purpose of analysis, the ocular findings in the acute and chronic stage were further classified as mild, moderate and severe according to an ocular severity score (OSS) at baseline and at 6 months. Mild ocular involvement had a score ranging from 1 to 4 , moderate ocular involvement had a score ranging from 5 to 8 , and severe ocular involvement had a score of $>8$. All the patients were stabilized by general supportive measures and all non essential drugs were discontinued. Systemic steroids were administered to selected patients with SJS and impending TEN who presented within 48 hours of onset of symptoms. A skin biopsy was performed whenever the clinical features were atypical and diagnosis based on clinical features alone could not be reached. Ocular medications included topical antibiotic and steroid eye drops and eye ointment, forniceal sweep of inflammatory adhesions when present was done to prevent symblepharon formation.

The data was analyzed as follows: first, the descriptive statistics were computed. These included the range, mean and standard deviation for quantitative variables, and category frequency counts and percentages for qualitative variables. Inferential statistics was then done using univariate and multivariate analysis. Pearson correlation was done to test the correlation between two continuous variables like age and OSSs and between OSSs. Analysis of variance was performed to test the association of categorical variables like dermatological diagnoses with the occurrence of ocular complications. Multivariate analysis was done using linear regression. Statistical significance was considered when $p$-value was $\leq 0.05$. All tests were done using SPSS ver. 16.0 (SPSS Inc., Chicago, IL, USA).

\section{Results}

Mean age was $28.15 \pm 15.78$ years. Thirteen of $44(29.9 \%)$ patients were of pediatric age group ( $\leq 18$ years) and $31 / 44$ (70.1\%) patients were adults. Out of the 44 patients, six patients who had no ocular involvement were excluded from the analysis of ocular manifestations of acute SJS/TEN. Out of the remaining 38 patients, two patients (5\%) were lost to follow up (i.e., not having attended at least one outpatient review) and three succumbed to the illness in the acute stage. These five patients were also excluded from the final analysis. Sixty-five eyes of 33 patients were subjected to analysis. One eye was excluded from analysis as there was previous history of herpetic keratouveitis and fungal corneal ulcer. There was no correlation between age and acute OSS; however, there was a statistically significant correlation between age of the patient and OSS at 6 months $(p=0.02)$. Younger age group had higher chronic OSS. There were 20 / 44 (45\%) male patients and 24 / 44 (55\%) female patients. There was no correlation between gender of the patient and severity of acute/chronic ocular involvement ( $p=0.72$ for males and $p=0.63$ for females). Thirty (68.2\%) patients had a diagnosis of SJS and 14 $(31.8 \%)$ patients had a diagnosis of TEN. Patients with TEN (the more severe disease of the two) had higher acute ( $p<$ $0.001)$ and chronic $(p=0.001)$ OSSs than SJS (Table 1).

Of the 44 patients, drugs were the most commonly associated causative factor in 42 patients $(95.4 \%)$. In the remaining two patients, the precipitating factor was not known. The causative drugs were anticonvulsants in 21 (45\%) patients, antibiotics in $17(36 \%)$ patients, anti-retroviral therapy in three $(6 \%)$ patients, nonsteroidal anti-inflammatory drugs in four (9\%) patients and others in two (4\%) patients (antifungal, oral contraceptive, and theophylline). Carbamazepine was the most common anticonvulsant implicated and in the antibiotic group it was sulfonamides. The number of drugs exceeds the total number of patients as some patients had used more than one drug in the period preceding the onset of symptoms. The period between drug intake and symptom onset (symptom lag) ranged from 1 to 41 days with a mean ( \pm standard devia- 
tion) of $13.33( \pm 8.33)$ days. The period between onset of symptoms and presentation to the hospital (presentation lag) ranged from 0 to 12 days with a mean ( \pm standard deviation) of $3.75( \pm 2.71)$ days. The time period from drug intake to onset of symptoms (symptom lag) showed a statistically significant correlation with both acute and chronic OSS ( $p=0.01, p=0.001$, respectively). Shorter time to onset of symptoms following drug intake correlated with higher grade of severity of ocular involvement. However, the time period between symptoms onset and presentation (presentation lag) to the hospital showed no correlation with the severity of acute or chronic ocular manifestations ( $p=0.785$ and $p=0.851$ respectively).

Table 1. Correlation of severity of dermatological manifestation with acute and chronic OSSs

\begin{tabular}{lccc}
\hline & $\begin{array}{c}\text { Dermatological } \\
\text { diagnosis }\end{array}$ & Mean of OSS & $p$-value \\
\hline OSS0 & SJS & $4.46 \pm 2.47$ & 0.001 \\
& TEN & $7.42 \pm 1.98$ & \\
OSS6 & SJS & $1.24 \pm 1.97$ & 0.001 \\
& TEN & $3.26 \pm 2.6$ & \\
\hline
\end{tabular}

OSS = ocular severity score; SJS = Stevens-Johnson syndrome; $\mathrm{TEN}=$ toxic epidermal necrolysis.
All patients (100\%) had oral mucosal involvement, 38 / $44(86.3 \%)$ patients had ocular involvement and $28 / 44$ $(63.6 \%)$ had genitalia involvement. Thirteen out of 33 patients had only two mucosal surface involvement while the remaining 20 / 33 had three mucosal surface involvement. Three mucosal surface involvement was associated with higher acute and chronic OSS $(p<0.001)$. Ten out of 44 $(22.72 \%)$ patients had human immunodeficiency virus (HIV) infection. There was no correlation between presence of HIV infection and severity of acute ocular involvement ( $p=0.11)$. However, there was a trend towards late ocular complications $(p=0.05)$. There were three $(6.8 \%)$ deaths during the acute phase of the disease. All three had a diagnosis of TEN. Severity of ocular manifestations in acute and chronic stage are shown in Table 2 and 3. During acute phase mild ocular involvement (OSS, 1 to 4) was seen in 28 / 65 (43.1\%) eyes, moderate ocular involvement (OSS, 5 to 8) was seen in 29 / 65 (44.6\%) eyes, severe ocular involvement (OSS >8) was seen in $8 / 65$ (12.3\%) eyes. There were no long-term ocular complications observed in 27 / 65 (41.5\%) eyes. At 6 months, mild ocular sequelae (OSS6, 1 to 4) was seen in $29 / 65$ (44.6\%) eyes. These comprised of mild lid margin irregularity and mild corneal staining. Moderate severity grade ocular sequelae (OSS6, 5 to 8$)$ were seen in $5 / 65(7.7 \%)$ eyes. These comprised of

Table 2. Severity grading of ocular manifestations in the acute stage of Stevens-Johnson syndrome and toxic epidermal necrolysis

\begin{tabular}{lcccc}
\hline Severity grade & Conjunctival hyperemia & $\begin{array}{c}\text { Conjunctival } \\
\text { inflammatory adhesion }\end{array}$ & Lid margin involvement & Corneal staining \\
\hline 0 & $0(0)$ & $26(40)$ & $11(16.9)$ & $24(36.9)$ \\
1 & $12(18.5)$ & $19(29.2)$ & $33(50.8)$ & $11(16.9)$ \\
2 & $41(63.1)$ & $18(27.7)$ & $16(24.6)$ & $26(40)$ \\
3 & $12(18.4)$ & $2(3.1)$ & $5(7.7)$ & $4(6.2)$ \\
\hline
\end{tabular}

Values are presented as number (\%).

Table 3. Severity grading of ocular manifestations at 6-month follow-up

\begin{tabular}{lccccc}
\hline Severity grade & $\begin{array}{c}\text { Conjunctival } \\
\text { hyperemia }\end{array}$ & $\begin{array}{c}\text { Lid margin } \\
\text { involvement }\end{array}$ & Corneal staining & Corneal opacification & $\begin{array}{c}\text { Corneal } \\
\text { neovascularization }\end{array}$ \\
\hline 0 & $51(78.5)$ & $45(69.2)$ & $29(44.6)$ & $59(90.8)$ & $69(92)$ \\
1 & $9(13.8)$ & $16(24.6)$ & $26(40)$ & $6(7.2)$ & $5(6.7)$ \\
2 & $3(4.6)$ & $4(6.2)$ & $10(15.4)$ & $0(0)$ & $1(1.3)$ \\
3 & $2(3.1)$ & $0(0)$ & $0(0)$ & $0(0)$ & $0(0)$ \\
\hline
\end{tabular}

Values are presented as number $(\%)$. 


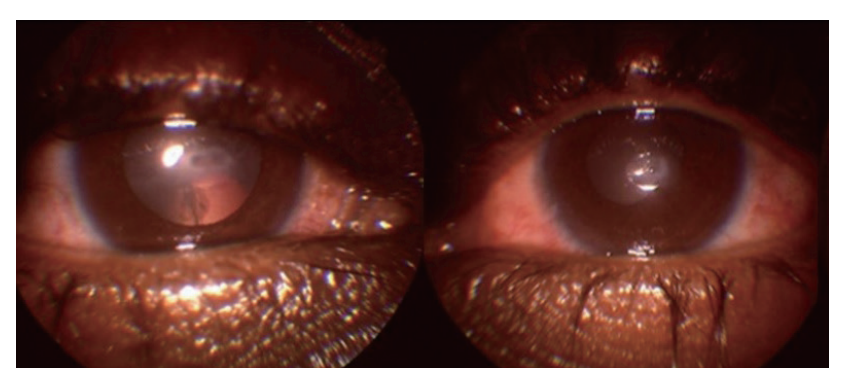

Fig. 3. Bilateral corneal perforation. Informed consent for publication of the clinical images was obtained from the patient.

grade 2 irregularity of lid margin, grade 2 corneal staining, meibomian gland involvement, and grade 1corneal opacities. Eight (10.6\%) eyes had meibomian gland involvement and none $(0 \%)$ had punctal occlusion by scarring. One patient had trichiasis. Severe ocular sequelae at 6-month follow-up (OSS6 >8) were seen in 4 / 65 (6.2\%) eyes of two patients. Out of two patients with severe ocular sequelae at 6 months, one patient was child with complications such as corneal neovascularization, opacification and extensive punctate corneal staining and other patient was with HIV infection, had bilateral severe dry eyes with bilateral corneal ulcers. He was managed initially for the corneal ulcers with antibiotics and intensive lubricants. In spite of this treatment, he developed bilateral corneal perforations. A trial of intramuscular vitamin A injection was given according to the recommended adult dosage and regimen. There was dramatic improvement following vitamin A administration with healing of corneal perforations (Fig. 3). We are unable to comment if the perforated corneal ulcers were a sequelae of SJS induced severe dry eye alone or if they occurred as a result of dry eye due to vitamin A deficiency associated with HIV infection. In 34 / 65 (52.3\%) eyes TFBUT was $<10$ seconds. Fourteen out of 63 (22\%) eyes had a basal Schirmer test result of $<10 \mathrm{~mm}$. Test could not be performed in $2 / 65$ eyes in a young child. Vital staining with rose bengal was positive only in $2 / 63$ (3.2\%) eyes. Two out of 65 eyes could not be assessed in a young child. Symptoms and signs suggestive of dry eye necessitating the continued use of lubricating eye drops at 6 months was seen in 23 / 65 (35.4\%) eyes. The acute OSS was found to correlate highly significantly with the chronic OSS, both at 1-month $(p=0.001)$ and at 6 -month ( $p=$ 0.001) follow-up.

Lid margin abnormality at presentation and corneal staining at baseline were found to be a very good predictor of chronic ocular complications both on univariate and multivariate analysis. Inflammatory adhesions at baseline showed a statistically significant association with ocular complications on univariate analysis. However, this effect did not persist on multivariate analysis.

\section{Discussion}

Ocular involvement in SJS/TEN in the acute stage is seen in $>50 \%$ of patients [3]. However, most patients are seen by ophthalmologists in the chronic stage as attention is directed towards maintenance of vital signs in acute stage and thus ocular management is often compromised. In this study, we found that younger age group patients were more likely to have long term ocular complications. This observation could be explained by the fact that there were more TEN patients in the younger age group. In the pediatric group, 6 / 13 cases had TEN and the remaining $7 / 13$ had SJS. We did not find any correlation between gender of the patient and severity of acute and chronic ocular complication. This study established that severity of dermatological disease correlated with more severe acute and chronic ocular involvement. This is different from other studies [5,6] where there was no such correlation. Although there was a trend to slightly higher late ocular complications in TEN in the study by Yip et al. [6], it was not statistically significant. They attributed this increased trend towards late ocular complications in TEN to the similar mechanisms of apoptosis occurring in the skin and eyes in TEN in contrast to SJS. The Fas-Fas ligand system is expressed in the cornea and overexpression of CD95 receptors in ocular tissue during TEN may play a role in the observed increased trend towards late ocular complications [6].

Drugs were identified as causative factor in majority of the cases. Anticonvulsants were the most common group of drugs implicated with Carbamazepine being the most common implicated drug, prescribed for both neurological and psychiatric patients [7]. Seven patients gave history of carbamazepine intake for treatment of neuralgia especially related to diabetic neuropathy in our study. The second most common group of drugs implicated was antibiotics, among which sulfonamides were the most common. In a study by Chang et al. [3], anticonvulsants were the most common triggers with carbamazepine being the leading 
cause. In other studies $[2,5,8]$ antibiotics were the most common agents implicated with sulfonamides being the most common group. There was a statistically significant correlation seen between symptom lag (time lag between intake of implicated drug and onset of symptoms) and severity of ocular manifestations in this study. The possible explanation to this observation could be that the hypersensitivity reaction to the drug in these patients was acute and was therefore more severe than those patients who took a longer time to manifest the drug hypersensitivity. There was no correlation seen between presentation lag and severity of ocular complications. This could be due to the fact that only those patients who presented within 2 weeks of onset of symptoms (in the acute phase of the illness) were included in the study and the necessary ophthalmological evaluation and treatment measures were implemented immediately on presentation. It would be expected that the occurrence of ocular sequelae would be higher in those patients who took a longer time to present to the hospital. We had two such patients with SJS who presented more than a month after onset of symptoms who are not included in this analysis; one patient had symblepharon and punctal scarring in both eyes; the other had bilateral severe dry eye with left corneal ulcer. The presence of more diffuse mucous and cutaneous damage may be associated with a higher risk of damage to the ocular mucous membrane [9].

The involvement of more than two mucosal surfaces showed a correlation with increased severity of both acute and chronic ocular involvement in this study $(p=0.001)$. Yip et al. [6] found no such correlation between the number of mucosal surfaces involved and the late ocular complications. The presence of HIV infection did not correlate with severe ocular involvement in the acute stage of SJS/ TEN. However, there was a trend towards increased ocular sequelae at the end of 6 months. The incidence of ocular involvement in this study was $86.4 \%$ (38/44 patients). Other studies have quoted varying incidences of $74 \%$ [9], $69 \%$ [6], 100\% [1]. The acute ocular involvement was mild in $43.1 \%$, moderate in $44.6 \%$ and severe in $12.3 \%$ (ocular involvement in acute stage was mild, moderate, severe in $58 \%, 8 \%, 8 \%$, respectively and $40 \%, 25 \%$, and $4 \%$, respectively in other studies) [6,9]. Our results are not comparable with other studies as we did not include the eyes with no ocular involvement for analysis. More importantly, we used two different grading systems for acute and chronic ocular manifestations unlike other studies [6,9] which have used the grading system developed by Power et al. [8]. In the severity grading of acute ocular involvement developed by Power et al. [8], we found that there is an overlap between severe ocular manifestations in acute stage with ocular sequelae in the chronic stage. Moreover, as this was a prospective study, the ocular manifestations in the acute and chronic stage were documented systematically. This could possibly account for the higher percentage of moderate and severe ocular manifestations in this study. In this study, the initial severity of acute ocular involvement correlated with the severity of chronic ocular findings. This correlation was also observed in another study [9]. However as five patients in the same study developed dry eye syndrome without initial ocular involvement, they concluded that late complications may also develop in patients without ocular symptoms at initial evaluation. Six patients had no ocular involvement in the acute stage in our study. These patients were lost to follow-up subsequently. Therefore, we cannot comment whether they developed any late ocular complication or not. However, Yip et al. [6] concluded that the severity of acute ocular complications does not predict late complications

When a univariate analysis was done to evaluate correlation between each of the components of the OSS at baseline with that at 6 months, it was found that there was a statistically significant correlation between severity grades of each of the components (conjunctival inflammatory adhesions, corneal involvement, lid margin involvement) with severity of chronic ocular complications. Multivariate analysis showed that severity of corneal and lid margin involvement in the acute phase showed significant correlation with severity of chronic ocular findings ( $p=$ $0.001)$ at 6 months. However, severity of conjunctival inflammatory adhesions showed no correlation with OSS at 6 months. This could be attributed to the following reasons: severe lid margin involvement with the attendant sequelae of lid margin irregularity could contribute to tear film instability, decreased TFBUT, dry eye manifesting as punctate corneal staining contributing to the overall increased chronic OSS. Secondly, corneal epithelial defects in the acute stage, classified as grade 3 corneal epithelial lesions could be a result of severe lid margin involvement with corneal exposure. On the other hand, corneal inflammation can occur independently in the acute stage, not caused by, but occurring simultaneously with lid margin/ 
tarsus inflammation. In a study by Di Pascuale et al. [10], there was no correlation between symblepharon and corneal complications in chronic stage. However, they found a significant correlation between corneal complications and eyelid cicatricial pathologies in the chronic stage.

There is no universally accepted treatment regimen for SJS and TEN and treatment with systemic corticosteroids remains controversial. There is also no standardized ophthalmologic treatment for prevention of ocular complications. However, topical steroids are an accepted modality of treatment of acute SJS/TEN as mentioned in literature $[1,3]$. In our study, a uniform treatment protocol with topical steroids, lubricants, antibiotics was instituted in all patients. Although there was no control group in our study, it was clear that the ocular treatment instituted comprising of topical steroids, preservative free lubricants, lysis of inflammatory adhesions with glass rod etc was beneficial. There were no chronic ocular complications noted in 27 / $65(41.5 \%)$ eyes. Symptoms and signs suggestive of dry eye necessitating the use of lubricating eye drops were seen in 23 / 65 (35.4\%) eyes. Only two out of 33 patients had major sight threatening ocular complications as previously mentioned. Best-corrected visual acuity was $6 / 6$ or better in 47 / 59 (79.7\%) eyes, <6 / 60 in 1 / 59 (1.7\%) eyes.

Sotozono et al. [1] structured interviews and analysis of hospital records of 94 patients showed that visual outcomes were significantly better in the group that received topical steroids at the acute stage (first week from disease onset) compared with those of the no treatment group ( $p<$ 0.001). In a retrospective study Chang et al. [3] reported that although the efficacy of early topical treatments for acute ocular manifestations of erythema multiforme/SJS/ TEN have not been evaluated in a prospective study, the authors believed that early management helps to reduce inflammation and destruction of the ocular surface and thus can minimize risk of long-term ocular sequelae. Treatment with systemic steroids remains controversial. In a retrospective review of hospital records of 366 patients with erythema multiforme/SJS/TEN it was concluded that systemic steroids used during the acute phase of the disease had no effect on the incidence or the severity of ocular manifestations [8]. As this is a retrospective study, the basis for selection of patients for systemic steroid therapy is not clear. Araki et al. [11] studied five patients with acute SJS/TEN who were treated with intravenous pulse therapy with methyl prednisolone along with topical steroids with- in 4 days of disease onset. All five patients had moderate to severe acute ocular manifestations. Skin eruptions dramatically improved after steroid pulse therapy. Ocular inflammation took a longer time to resolve with regeneration of conjunctival and corneal epithelium by 6 weeks. Visual acuity and ocular findings at the chronic stage were evaluated after 1 year from disease onset. In all eyes best-corrected visual acuity at 1 year from disease onset was 20 / 20 or better. There were no major ocular sequelae noted. It was concluded that steroid pulse therapy at disease onset is of great therapeutic importance in preventing ocular complications. Also, topical betamethasone may be helpful in preventing corneal epithelial stem cell loss and cicatricial changes [11]. However, the number of cases is too small to know with certainty whether the self-limiting nature of the disease or whether the institution of systemic steroid therapy was responsible for the promising results. Effect of systemic steroids on acute and chronic ocular complications was not evaluated in our study as only select patients received systemic steroids as per the discretion of the dermatologist.

We would like to emphasize the use of preservative free eye drops in the acute stage of ocular involvement in SJS/ TEN as preservatives in eye drops are known to cause ocular surface damage. Ocular damage such as acute and delayed hypersensitivity, stromal and endothelial cell edema by preservative has been demonstrated in a study. Yip et al. [6] found that topical antibiotic use in the acute phase correlated with long term ocular complications. They attributed this finding to ocular surface damage by preservatives in antibiotic eye drops as well as direct toxicity to the accessory lacrimal glands by the topical antibiotic itself. It was concluded in another study as well that topical antibiotics and other topical formulations that can lead to severe dry eyes should be used with care [9]. Topical antibiotic cover at minimum frequency was part of our treatment protocol. Tobramycin (an aminoglycoside antibiotic) was used and fluoroquinolones were avoided as oral fluoroquinolones have been associated with SJS [12]. Non-steroidal anti-inflammatory drops were avoided as well, as their use has been associated with severe corneal complications in SJS [13].

Our study has several limitations. There is a possibility of under-estimating long term ocular complications because of the small sample size and short duration of follow-up. These patients need to be followed up for at least 
1 year to assess for ocular sequelae. We do not have control group for comparison as all patients received the same ophthalmic treatment. Also, we have studied the effect of only the medical ocular management for acute SJS/TEN. Surgical management of acute SJS/TEN with amniotic membrane grafting and subsequent ocular sequelae needs to be studied. In conclusion, the younger age of the patient correlated with severity of long-term ocular complications. Greater severity of the disease such as a diagnosis of TEN, more number of mucosal surfaces involved and shorter symptom lag correlated with more severe acute and chronic ocular manifestations. The severity of lid margin involvement and corneal involvement in acute stage were good predictors of severity of chronic ocular findings. There was no correlation between presence of HIV infection and severity of initial ocular manifestations although there was a trend towards increased long term ocular complications. Intensive ocular treatment comprising topical corticosteroids, preservative free tears substitutes, lysis of conjunctival inflammatory adhesions and steroid-antibiotic eye ointment for local application over lid margin is safe and effective in reducing ocular surface inflammation in the acute stage. The overall ocular outcome was good in this study. Visual outcome at 6 months was good in all patients except one.

\section{Conflict of Interest}

No potential conflict of interest relevant to this article was reported.

\section{References}

1. Sotozono C, Ueta M, Koizumi N, et al. Diagnosis and treatment of Stevens-Johnson syndrome and toxic epidermal necrolysis with ocular complications. Ophthalmology 2009;116:685-90.

2. Kompella VB, Sangwan VS, Bansal AK, et al. Ophthalmic complications and management of Stevens-Johnson syndrome at a tertiary eye care centre in south India. Indian $J$
Ophthalmol 2002;50:283-6.

3. Chang YS, Huang FC, Tseng SH, et al. Erythema multiforme, Stevens-Johnson syndrome, and toxic epidermal necrolysis: acute ocular manifestations, causes, and management. Cornea 2007;26:123-9.

4. Sotozono C, Ang LP, Koizumi N, et al. New grading system for the evaluation of chronic ocular manifestations in patients with Stevens-Johnson syndrome. Ophthalmology 2007:114:1294-302.

5. van Bijsterveld OP. Diagnostic tests in the Sicca syndrome. Arch Ophthalmol 1969;82:10-4.

6. Yip LW, Thong BY, Lim J, et al. Ocular manifestations and complications of Stevens-Johnson syndrome and toxic epidermal necrolysis: an Asian series. Allergy 2007;62:527-31.

7. James J, Sushma M, Guido S, Elizabeth J. Cutaneous adverse drug reactions in a South Indian tertiary care center. Indian J Dermatol 2005;50:17-21.

8. Power WJ, Ghoraishi M, Merayo-Lloves J, et al. Analysis of the acute ophthalmic manifestations of the erythema multiforme/Stevens-Johnson syndrome/toxic epidermal necrolysis disease spectrum. Ophthalmology 1995;102:166976.

9. Gueudry J, Roujeau JC, Binaghi M, et al. Risk factors for the development of ocular complications of Stevens-Johnson syndrome and toxic epidermal necrolysis. Arch Dermatol 2009;145:157-62.

10. Di Pascuale MA, Espana EM, Liu DT, et al. Correlation of corneal complications with eyelid cicatricial pathologies in patients with Stevens-Johnson syndrome and toxic epidermal necrolysis syndrome. Ophthalmology 2005;112:904-12.

11. Araki Y, Sotozono C, Inatomi T, et al. Successful treatment of Stevens-Johnson syndrome with steroid pulse therapy at disease onset. Am J Ophthalmol 2009;147:1004-11.

12. Hallgren J, Tengvall-Linder M, Persson M, Wahlgren CF. Stevens-Johnson syndrome associated with ciprofloxacin: a review of adverse cutaneous events reported in Sweden as associated with this drug. J Am Acad Dermatol 2003;49(5 Suppl):S267-9.

13. Isawi H, Dhaliwal DK. Corneal melting and perforation in Stevens Johnson syndrome following topical bromfenac use. J Cataract Refract Surg 2007;33:1644-6. 